\title{
Análisis de la implementación de los programas de Educación Media Alternativa y a Distancia de la modalidad de jóvenes y adultos
}

\author{
Analysis of program implementation in Alternative Secondary \\ Education and Distance Education for Youth and Adults
}

\author{
María Victoria Zavala Saucedo \\ Graciela María Delia Molinas Santana \\ Hugo Matías Speratti Mendoza
}

\section{RESUMEN}

Este trabajo pretende dar un panorama sintético del Informe Final de Investigación, presentado al MEC en el mes de febrero del presente año.

El trabajo se ha centrado en un acercamiento a las ofertas educativas de la Educación Media Alternativa y a Distancia. El objetivo general del estudio es analizar el proceso de implementación curricular de la Educación Media Alternativa (EMAJA) y la Educación Media a Distancia para Jóvenes y Adultos (EMADJA).

Algunas conclusiones resaltantes son que las infraestructuras en la que se desarrollan las ofertas, en su mayoría se realizan en espacios utilizados para Educación Escolar Básica (EEB) y la Educación Inicial (EI), resultando inadecuados a la edad de los participantes. Se resalta la ausencia de un documento curricular oficial en EMAJA; en EMADJA, si bien se cuenta con este documento que declara el énfasis en Nuevas Tecnologías, las evidencias recogidas denotan la ausencia de medios tecnológicos. Las evidencias del proceso de implementación son escasas. Los docentes que se desempeñan en las mismas, cuentan con certificación para el desempeño docente además de compartir con los y las participantes la alta valoración social de la oferta que representa dignidad, posibilidades sociales y económicas.

Palabras clave: Educación Media, Educación a Distancia, Nuevas tecnología en la Educación, 


\section{ABSTRACT}

This report aims to provide a synthetic overview of the Research Final Report developed by MEC in February of this year.

The work focuses on looking closer at the educational opportunities in both Alternative Secondary Education and Distance Education. The general goal was to analyse the process of implementation in the Alternative Secondary Education (EMAJA) and Distance Education (EMADJA) syllabi for youth and adults.

Some conclusions showed that the infrastructures in which the opportunities are developed take place in Primary Education (EEB) and Preschool Education (EI), proving inadequate for the age of participants. It should be noticed that the absence of an official syllabus in EMAJA. Though EMADJA's syllabus has an emphasis in New Technologies, although observations show their lack of technological means. Evidence of the implementation process is limited. Teachers are certified to do the work, and in addition share with participants the high social value of the opportunities, which represent dignity and social and economic possibilities.

Keywords: Secondary education, Distance education, New technologies for education. 


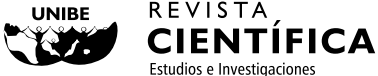

\section{INTRODUCCIÓN}

La Educación Media en el proceso de Reforma Educativa incorpora la implementación de las modalidades de Educación Media a Distancia para Jóvenes y Adultos, desde el año 2006, y la Educación Media Alternativa para Jóvenes y Adultos, desde el año 2007, en forma experimental. El Sistema Educativo Nacional implementa estas modalidades como una opción que tienen los jóvenes y adultos que aspiran a completar sus estudios.

Este trabajo de investigación llevado a cabo por la Universidad Iberoamericana, desde su Centro de Investigación, presenta los resultados del proceso de indagación sobre cómo se implementan las ofertas de formación de la Educación Media Alternativa y la Educación Media a Distancia de la modalidad para jóvenes y adultos.

Como hipótesis de trabajo se asumió que la falta de información dificulta el acompañamiento de la implementación del currículum.

A partir de la pregunta central se formularon los objetivos de la investigación.

El objetivo general de la investigación es analizar el proceso de implementación curricular de la Educación Media Alternativa y la Educación Media a Distancia de la modalidad para Jóvenes y Adultos. Asimismo se establecieron los objetivos específicos: contextualizar los centros educativos que implementan las modalidades de la Educación Media para Jóvenes y Adultos; describir el diseño curricular de la Educación Media Alternativa y la Educación Media a Distancia de la modalidad para Jóvenes y Adultos; determinar las características del proceso enseñanza-aprendizaje en estas dos ofertas; identificar la percepción de los actores involucrados en la implementación curricular de la Educación Media Alternativa y la Educación Media a Distancia de la modalidad para Jóvenes y Adultos.

La incorporación de la universidad en el trabajo de investigación se realizó a través de la presentación a una licitación pública convocada por el Ministerio de Educación y Cultura (MEC), la cual, cumpliendo los requisitos solicitados, fue adjudicada para la ejecución del estudio.

Cabe resaltar la colaboración destacada de las instituciones educativas, por la excelente predisposición para la entrega de documentaciones solicitadas y por la predisposición para donar sus tiempos destinados al desarrollo de las entrevistas con los actores involucrados. 


\section{METODOLOGÍA}

Para buscar respuesta al problema y lograr los objetivos establecidos, se decidió realizar una investigación de diseño no experimental, de tipo exploratorio y con enfoque cualitativo. La investigación se definió como un estudio de caso múltiple y se realizó en 16 (dieciséis) instituciones educativas que implementan las ofertas de Educación Media Alternativa y Educación Media a Distancia en la modalidad para Jóvenes y Adultos en la República del Paraguay, entre los meses de octubre y diciembre de 2010 .

Las instituciones que formaron parte del estudio fueron seleccionadas teniendo en cuenta los siguientes criterios: tipos de unidad operativa: institución de Media Alternativa y de Educación Media a Distancia; tipo de gestión: pública y privada; localización geográfica en los departamentos del país. Así también se tomaron en cuenta las recomendaciones de los referentes de la Dirección General de Educación Permanente del Ministerio de Educación y Cultura.

\section{Cuadro $N^{0} 1$ : Muestra estudiada}

\begin{tabular}{|c|c|c|c|c|c|c|}
\hline \multicolumn{2}{|c|}{ Instituciones } & Alumnos & Profesores & Coordi & Técnicos & Directores \\
\hline EMAJA & 6 & 64 & 16 & & 4 & 6 \\
\hline EMADJA & 10 & 82 & 31 & 10 & & \\
\hline
\end{tabular}

Por tanto, la muestra utilizada por la investigación fue de tipo intencional (Martínez, 1997) y por conveniencia (Sampieri, et.al., 2008), teniendo en cuenta que se trabajó con las instituciones que aceptaron formar parte del trabajo y quedó constituida con 16 instituciones educativas -diez de Educación Media a Distancia para Jóvenes y Adultos y seis de la Educación Media Alternativa para Jóvenes y Adultos- ubicadas en nueve departamentos geográficos de la República del Paraguay: Central, Alto Paraná, Boquerón, Caaguazú, Concepción, Itapúa, Misiones, Guairá y Paraguarí.

Para la recolección de los datos se emplearon dos técnicas: la entrevista y el análisis de documentos. Las entrevistas se aplicaron, en forma grupal a los docentes y tutores de los cursos, así como a los participantes 
y alumnos de los 16 centros educativos estudiados. Asimismo, se realizó la entrevista grupal con tres referentes técnicos y un coordinador de la Dirección General de Educación Permanente de Jóvenes y Adultos del MEC. Las entrevistas individuales se aplicaron a los coordinadores de sedes tutoriales de EMADJA y directores de centros de EMAJA.

El análisis de documentos se aplicó a los materiales proporcionados por las instituciones visitadas: documentos curriculares de la Educación Media a Distancia, módulos, planillas estadísticas, cuadro de personal de las instituciones, planilla de calificaciones y documento de evaluación de la Educación Media a Distancia.

Las entrevistas fueron aplicadas a los segmentos de actores en las mismas instituciones educativas. Se desarrollaron en ambas modalidades: individuales y grupales. Duraron aproximadamente una hora por cada segmento de actores.

La guía de análisis se aplicó a los documentos proveídos por las instituciones responsables.

El tratamiento de los datos se realizó por categorización emergente, convirtiendo los datos de la entrevista en texto, y analizando el contenido individualmente y por segmento, para identificar las concordancias, discordancias y lo atípico. Se codificaron y se reconocieron categorías de análisis que fueron transcribiéndose en un cuadro de sistematización de las evidencias. Esto facilitó la organización de la información y la obtención de una visión de conjunto por segmento de actores y por cada tipo de oferta, estableciéndose los hallazgos más significativos acordes a los objetivos del estudio.

\section{RESULTADOS}

Respecto de la Educación Media Alternativa, en cuanto a ContextoInfraestructura y Recursos Didácticos, se hallaron los siguientes datos: el espacio que disponen las instituciones está infantilizado pues es el mismo que se utiliza de día para la EI, la EEB y la EM. Asimismo, los alumnos no disponen de biblioteca, de cantina ni de sala de primeros auxilios, pues están cerradas por la noche. Sólo un profesor mencionó disponer de biblioteca. Los recursos didácticos con que cuentan son los tradicionales, como pizarra, libros, fotocopias y ejercitarios, además de computadoras, infocus y videos, que se mencionó en dos casos. Los profesores destacaron que elaboran ejercitarios para sus alumnos. 
Resaltó en los resultados que en la modalidad con énfasis en Nuevas Tecnologías no cuenta con computadoras ni sala de informática. Según los docentes, en algunos de los centros estudiados, "las computadoras con internet traen los estudiantes" (sic.).

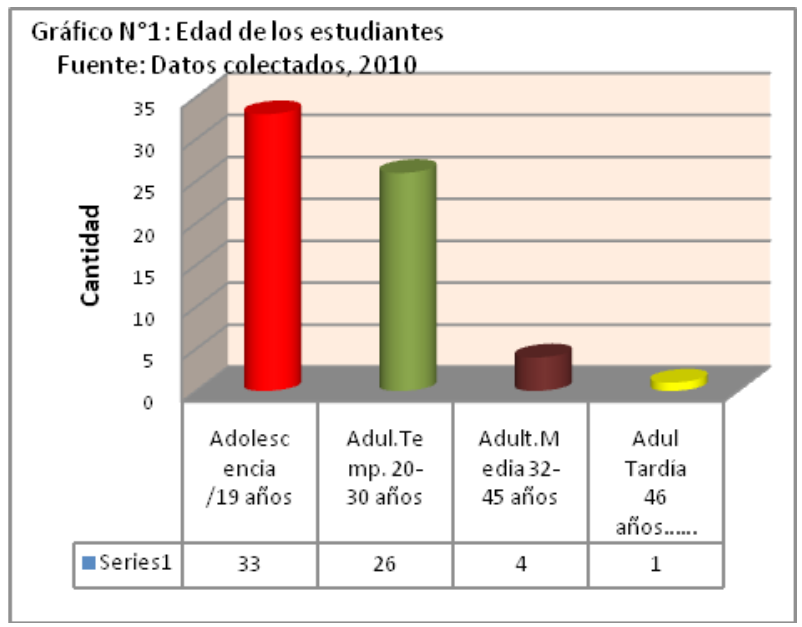

Respecto de la caracterización de los actores educativos, se destacaron el ausentismo y las llegadas tardías de los alumnos, las dificultades en el aprendizaje de matemática, física y química. Los docentes caracterizaron a sus estudiantes como trabajadores en diversas ocupaciones, excluidos del sistema regular debido a razones laborales, económicas, problemas familiares y otros.

Si bien los docentes declararon que los alumnos son trabajadores, estos mismos alumnos manifestaron no estar trabajando, lo cual podría indicar una tendencia a la generalización de algunos pocos casos. Asimismo, expresaron que los adultos tienen mayor disposición para aprender, demuestran más interés y empeño en los trabajos, pero tienen un aprendizaje más lento en relación con los más jóvenes.

Los estudiantes entrevistados son de sexo femenino en una amplia mayoría: $73 \%$, y en relación con la edad, el 50\% del total de los alumnos pertenece a la franja etaria de la adolescencia, tal como muestra el gráfico $\mathrm{N}^{\circ} 1$.

Los profesores también son en un 56\% de sexo femenino, y en cuanto a la edad, se reparten casi equitativamente un tercio cada franja: adultez temprana (jóvenes), adultez media y adultez tardía. En cuanto 
a coordinadores y directores, están ubicados en la adultez tardía conforme a la categorización de E. Erickson (López, 2002). Según los datos, se verifica que la mayoría de los docentes está en el proceso de consolidación de sus competencias en la enseñanza teniendo en cuenta los años de servicio en la docencia.

En lo referente al Diseño curricular, las características declaradas lo indican como currículum abierto, flexible, accesible, de aprendizaje permanente, con desarrollo de funciones cognitivas y profesionales. Consta de un Plan común y un Plan específico. El perfil de salida insiste en las funciones cognitivas y en las funciones profesionales. El documento no editado de la Dirección General de Educación Permanente señala que será capaz de la "interrelación social; para solucionar situaciones de la vida real aplicando fundamentos científicos para constituirse en ciudadano comprometiéndose con el desarrollo social; (...) y un productor de bienes y servicios cuyas utilidades favorecerán su desarrollo personal y el de la sociedad". En las funciones profesionales, también se menciona la necesidad de manejar las Nuevas tecnologías; sin embargo, este estudio constata que carecen de PC incluso las instituciones que desarrollan el Plan con énfasis en NTIC. En cuanto al manejo que los profesores tienen del currículum, se destacó que conocen el Plan y el listado de capacidades, pero se reconoce lo confuso que resulta la presentación de la malla. Los directores también reconocieron esta confusión y se constatan en el documento algunos vacíos en las intencionalidades pedagógicas. En cuanto a la evaluación, está clara en el peso: $50 \%$ del semestre: $30 \%$ proceso y $20 \%$ trabajos a distancia. Lo que resalta es que si bien el diseño menciona que se aplicarán las pruebas nacionales, los profesores en ningún momento mencionaron la aplicación de estas pruebas estandarizadas.

En relación con las experiencias de aprendizaje-enseñanza, los docentes no llegaron a caracterizar el planeamiento que hacen, sino más bien mencionaron los ejercitarios. Asimismo, no refirieron ni detallaron contenidos: se refieren a áreas o materias que trabajan, desarrollando el programa que viene del MEC, lo que muestra que se ocupan del "currículum entregado" según las expresiones de los docentes: "los programas son los enviados (por el MEC), pero si hay tiempo se agregan o se modifican de acuerdo a la necesidad".

Las estrategias son variadas: exposiciones, trabajos individuales, grupales, ejercitarios, proyectos, charlas. Los alumnos hacen mención a las mismas y agregan que son dinámicas y motivadoras. La evaluación 
se realiza con control de tareas y pruebas escritas, sólo dos profesores mencionaron: "a través de la Feria de Ciencias" y otro "con la técnica de licuadora"". Manifestaron que no hay claridad en las orientaciones técnicas de las instancias responsables del MEC para la elaboración de los instrumentos de evaluación y que el trámite de aprobación del test depende de la Supervisión Pedagógica. Sugirieron ajustar el sistema de evaluación y reconocen que sería adecuado bajar la exigencia del $70 \%$ al $60 \%$.

En cuanto a la Percepción de los actores respecto de la oferta, resaltó la falta de capacitación y seguimiento por parte del MEC; percibieron que los contenidos deben ajustarse, con un programa más detallado. Expresaron también que el MEC pone muchas trabas para esta modalidad, como si quisieran eliminarla del sistema, y que desde que existe PRODEPA la modalidad se "degeneró", decayó la cantidad de alumnos, se abrieron muchas instituciones paralelas y las reuniones que se realizan en Asunción son una dificultad por no haber un criterio común y por no haber un plan del MEC". Todos los actores expresaron su satisfacción por estar en esta modalidad y por ofrecer la oportunidad a los jóvenes y adultos de culminar sus estudios y poder seguir los superiores.

En lo referente a la Educación Media a Distancia para Jóvenes y Adultos, se halló en relación con la Infraestructura y Recursos didácticos que la modalidad a distancia se desarrolla con la presencia de los estudiantes en las sedes, lo cual evidencia un desajuste con relación a los requerimientos de la modalidad a distancia; tampoco cubre la necesidad de infraestructura para la permanencia de los alumnos. La carencia de recursos tecnológicos es evidente y claramente percibida por estudiantes y tutores que no sólo reconocen la escasísima cantidad de equipos, sino su obsolescencia.

Es de mencionar que la Res. MEC 35/07 no presenta en su texto la garantía para una educación con énfasis en las Nuevas Tecnología, pues sólo exige como requerimiento para el funcionamiento de la oferta "una computadora".

1 Técnica utilizada para dinamizar los encuentros que consiste en que todos los participantes estarán tomados de las manos haciendo un círculo, mirándose unos a los otros. Uno de los integrantes es el que va a llevar a cabo el siguiente procedimiento: este integrante, sin soltar las manos de sus compañeros de al lado, se meterá por debajo de las manos de los jóvenes que se encuentren frente a él; jalando consigo los demás, hasta que todos estén totalmente volteados de espalda haciendo el circulo. No se permite que los integrantes se suelten en ningún momento; porque el que inició el juego deberá jalar a todos haciendo girar el círculo lo más rápido posible sin que los integrantes se suelten. 
En cuanto a los actores educativos, los resultados muestran que los tutores tienen preparación y están dentro de la adultez temprana. Los estudiantes, en un 55\% son adolescentes -como puede verse en el gráficoen condiciones etarias de insertarse en el Sistema en la Educación Media Regular; sin embargo, la flexibilidad y el horario los atraen. Es de resaltar que el 55\% de los estudiantes investigados pertenecen al sexo femenino.

\section{Gráfico $\mathbf{N}^{\circ}$ 2: Edad de los participantes EMADJA}

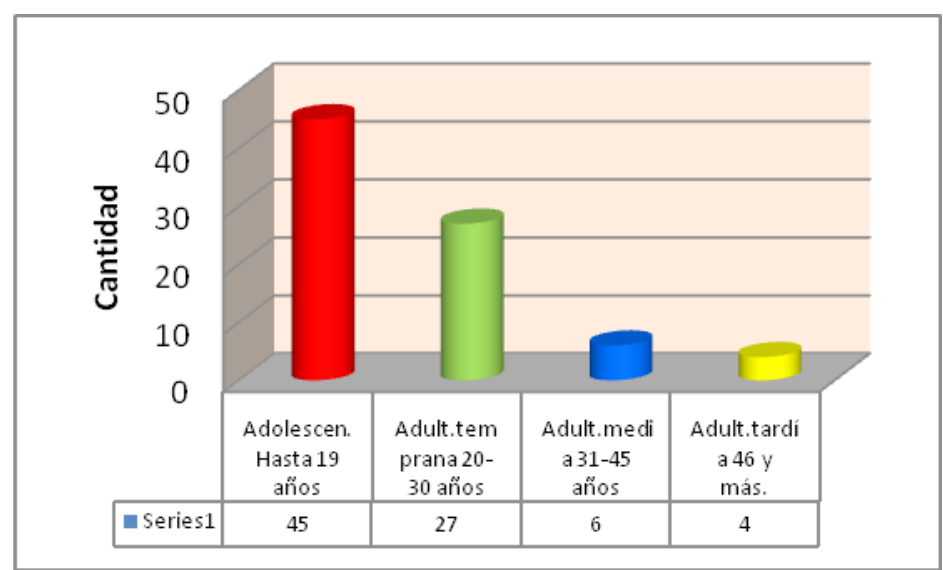

Otra característica señalada es que no existe un seguimiento sistemático a los participantes. De igual modo, si bien los alumnos concurren asiduamente a la escuela, los coordinadores manifestaron que a las tutorías asisten solo el $50 \%$ del tiempo requerido. Respecto de los tutores, en cuanto a la formación académica, cuentan con el perfil requerido para la enseñanza en la modalidad, y con el título específico conforme al ámbito en el que se desempeñan. Lo mismo ocurre con los coordinadores y los técnicos.

Respecto del diseño curricular, los datos indicaron que: el curriculum declarado es abierto y flexible, con aprendizajes pertinentes y relevantes que orienten a la autosuficiencia, con metodología accesible y personalizada, con sistema modular y por ámbitos de conocimientos. También se halló que en la realidad, la competencia "digital" que forma parte del perfil de salida se ve obstruida en su desarrollo por la carencia de equipos informáticos. Asimismo, otra dificultad constituye que aun contando con algunos equipos, no se tiene acceso a Internet, lo cual desvirtúa el desarrollo del último modulo de la cursada que plantea una modalidad totalmente virtual. 
En lo que respecta a las experiencias de enseñanza-aprendizaje, declararon que por la modalidad no planifican y la secuenciación de contenidos supliría al planeamiento. Es reiterativa la expresión de que se basan en el libro guía tal como lo señalaron: "No hay una planificación, debido a las características de la oferta y las actividades de la tutoría son desarrolladas conforme a lo planteado en el libro". No se refieren a contenidos -salvo los de informática-, sino a materias, y refirieron que los contenidos son "adecuados al nivel", "pertinentes con base en capacidades", y "son menos que la media regular". En cuanto a las estrategias que aplican, están las demostrativas, explicativas, personales y control de tareas. Asimismo, los tutores realizan sus funciones vía correo electrónico, si fuera posible, y si no, por teléfono.

Respecto a la evaluación, resalta que todos los test deben ser aprobados por la Supervisión Pedagógica, lo cual, por la distancia, lo convierte en un trámite muy engorroso. Asimismo, algunas sedes deben trasladarse a otras para el día de la evaluación a fin de poder usar los equipos y tener acceso a Internet. Un dato llamativo se encuentra en las expresiones de los coordinadores: "La asistencia es irregular, por tanto, hay que hacer tutorías individuales".

Sobre la percepción de las ofertas, todos los actores de las sedes - excepto los de una de ellas-, coinciden en que el equipamiento es precario, insuficiente y obsoleto. Expresaron su necesidad del siguiente modo: "necesitamos mejor infraestructura", "un espacio adecuado y ventilación", "el espacio es pequeño y el local es prestado, por lo que se requieren sillas adecuadas", es una "modalidad preparada para funcionar con Internet, pero la conexión solo hubo al inicio, luego se cortó", "por la falta de conexión el desarrollo del cuarto módulo, sobre todo, se hace difícil". Esta referencia al cuarto módulo es reiterativa: se indica desarrollarlo en la plataforma, pero no hay internet, una coordinadora sugirió incluso suprimirlo de la plataforma, pues no es real y darlo comúnmente, con escritos.

No obstante, se sienten satisfechos de trabajar en esta modalidad, reconocen que es útil para los estudiantes y manifiestan agrado por los kits de comienzo de año y el material entregado. Los participantes valoraron la oferta expresando su utilidad para "continuar los estudios y como una buena oportunidad para que la gente pueda terminar su bachillerato y conseguir un buen empleo.

Asimismo, expresaron que sienten la necesidad de estar más acompañados por el MEC manifestando que "no se acompaña, se siente como si fuera un parásito del colegio", "sólo al inicio de la modalidad hubo capacitación". 


\section{$=$

\section{DISCUSIÓN}

A continuación se presentan las siguientes ideas a partir de las interrogantes planteadas para desarrollar la investigación y que se constituyeron en objetivos de la misma.

La pregunta inicial fue ¿cómo se realiza el proceso de implementación curricular de la Educación Media Alternativa y la Educación Media a Distancia de la modalidad para Jóvenes y Adultos? Para dar respuesta a esta interrogante, se recurrió a las siguientes: ¿cómo es el contexto en el cual se desarrollan estas ofertas educativas?, ¿cuáles son las características de los diseños curriculares?, ¿cómo se da el proceso de enseñanza y aprendizaje? y ¿cómo perciben los actores educativos la implementación de las ofertas?

En relación con el contexto infraestructura, en la EMAJA, en las seis instituciones visitadas los datos evidencian que los espacios destinados para el funcionamiento están preparados para otras ofertas educativas del Sistema Educativo Nacional, por lo que resultan poco propicias las condiciones que se les ofrecen a los y las estudiantes que son jóvenes y adultos. Cabe preguntarse: ¿cuáles son los supuestos que operan en la decisión de que los espacios destinados para niños y niñas serían los apropiados para la franja etaria a la cual está destinada esta oferta?. La respuesta a esta pregunta propicia el análisis sobre la influencia de las condiciones como un factor de relevancia para el desarrollo de proceso de aprendizajes.

En la Educación Media a Distancia para Jóvenes y Adultos, en las diez instituciones visitadas se evidencia una situación similar, con algunas variantes encontradas en tres Centros de Recursos de Educación Permanente que presentan condiciones para la atención de los y las participantes; cabe resaltar que estas instituciones solo están destinadas para impartir ofertas educativas para jóvenes y adultos.

En las dos modalidades investigadas, los datos indican que recursos didácticos son escasos y de difícil acceso, como es el caso de las bibliotecas. La situación en la Modalidad a Distancia es crítica considerando que solo en una de las instituciones se pudo comprobar que los participantes del último módulo de la oferta educativa tienen acceso a medios informáticos e Internet, sobre todo porque la oferta se presenta con el énfasis en Nuevas Tecnologías; las demás instituciones visitadas no cuentan con dichos recursos. Estas evidencias presentan la necesidad 
de una revisión profunda de la pertinencia de la oferta curricular ante las condiciones reales en las que se implementan estas modalidades educativas del nivel medio.

En relación con los diseños curriculares, los datos indican la necesidad de diseños curriculares pertinentes al desarrollo de las competencias y capacidades definidas en el perfil de salida de la Educación Media, que es igual para todas las modalidades ofrecidas en este nivel del Sistema Educativo Nacional. Además, cabe resaltar que el diseño curricular no es solo un documento más, es necesario atender el proceso de implementación del currículum, que, según Gimeno Sacristán (2002), dicho proceso, "cruza por un complejo entramado de prácticas en un proceso de desarrollo en diferentes niveles de concreción y objetivación". Así, el autor plantea el siguiente análisis sobre esos procesos:

a) El currículum prescripto es el primer nivel de definición y regulación político-administrativa y es considerado básico y obligatorio.

b) El currículum presentado a los profesores se demuestra a través de "una serie de medios, elaborados por diferentes instancias que suelen traducir el significado y los contenidos del currículum".

c) El currículum "moldeado por los profesores", es el nivel por el cual el currículum pasa por el "tamiz de la interpretación de los profesionales de la enseñanza".

d) El currículum en acción es la práctica concreta y real que gerencia el docente en interacción con sus alumnos; esto lo realiza a través de "las tareas académicas, que a modo de elementos morales vertebran lo que es la acción pedagógica, donde podemos apreciar el significado real de lo que son las propuestas curriculares".

e) El currículum realizado se presenta como consecuencia de la práctica concreta llevada a cabo en interacción entre docentes y estudiantes en un contexto específico; "son efectos a los que unas veces se les presta atención porque son considerados 'rendimientos' valiosos y prominentes del sistema o de los métodos pedagógicos".

f) El currículum evaluado se refiere a los contenidos enseñados que los profesores seleccionan y exigen en la comprobación de los saberes de los exámenes a los cuales los estudiantes se someten, además incluye el cómo lo exigen y cómo lo valoran. 
Vinculando lo expuesto por Sacristán (2002) y las evidencias recogidas, se puede ver que el primer nivel propuesto por el autor no está aún definido en la EMAJA. Cabe resaltar que para las dos modalidades, es necesario desarrollar dispositivos que propicien el seguimiento de los demás niveles de implementación presentados por el autor citado.

En las dos modalidades en estudio, los datos recogidos evidencian que los procesos de enseñanza y aprendizaje no superan la explicación del docente y la elaboración de ejercicios, dejando de lado principios propuestos por la andrología como el de centrarse en la características de los adultos.

En relación con la percepción de las dos modalidades, es importante identificar quiénes son los actores educativos que han manifestado sus opiniones.

Los datos recogidos en relación con los estudiantes indican que es poco el tiempo que dejaron el sistema regular y se reinsertaron al sistema educativo a través de estas modalidades; se percibe el valor que los jóvenes otorgan a la educación media, sobre todo porque retoman luego de haberla abandonado. Las razones podrían estar originadas por los requerimientos que se les presentan para incorporarse en el ámbito laboral o para proseguir con sus estudios en el nivel de la Educación Superior; cualquiera de las razones son relevantes para asegurar el acceso a la educación como derecho y aspiración de una sociedad que pretende mejorar las condiciones de vida de sus miembros. Además, se constituye en una oportunidad para el desarrollo de aprendizajes pertinentes considerando el interés asumido al retomar los estudios.

En cuanto a los docentes, es relevante que todos y todas se encuentran certificados. Cabe identificar la preparación con la que cuentan para atender a jóvenes y adultos, considerando que si en su formación se obvió el estudio de la andragogía y se centró en la pedagogía, estarán más preparados para atender a niños y niñas, no así a adultos o jóvenes con decisiones adultas. Este es un aspecto esencial a ser tenido en cuenta en la formación continua de estos docentes ya que este aspecto puede que origine un trato infantilizado, y uniendo este aspecto con el señalado en relación con el contexto, es más complejo aún para lograr pertinencia de la oferta educativa.

Los datos evidencian coincidencias entre los actores consultados; por un lado, participantes así como docentes, coordinadores y directores identifican las limitaciones de las condiciones de la infraestructura y de los recursos didácticos, pero valoran más la posibilidad de la certificación 
del bachillerato. Esta valoración es un aspecto positivo para incorporar innovaciones que mejoren la oferta educativa con políticas que atiendan las limitaciones señaladas; por el otro lado, los docentes, coordinadores y directores la necesidad de estar más acompañados por el MEC están dispuestos a acompañar los procesos de seguimiento y monitoreo de los diferentes niveles de concreción del currículum de las ofertas educativas estudiadas en esta investigación.

Se puede señalar que el análisis del proceso de implementación curricular de la Educación Media Alternativa (EMAJA) y la Educación Media a Distancia para Jóvenes y Adultos (EMADJA) presenta la necesidad de ser revisados y resignificados a partir de los procesos de implementación curricular que plantea el marco teórico y las posibilidades reales de los mecanismos desplegados por MEC en los procesos de gestión de la implementación de las ofertas educativas.

\section{CONCLUSIÓN}

\section{CONTEXTO, INFRAESTRUCTURA Y RECURSOS DIDÁCTICOS}

La EMAJA ofrece a los estudiantes espacios infantilizados pues son los mismos que utilizan los de la EEB y la EI. Los alumnos no disponen de los servicios básicos (biblioteca, cantina, primeros auxilios). Los recursos didácticos con que cuentan son tradicionales: libros, cuadernos, pizarra, fotocopias y ejercitarios. Es contradictorio que en la EMAJA con énfasis en Nuevas Tecnologías no se cuente con computadoras ni salas de informática. Este aspecto se reitera en la EMADJA, en la cual los equipos son insuficientes, obsoletos y sin mantenimiento, haciendo que los alumnos deban trasladarse a otros centros para rendir los exámenes finales en las PC -sin haber realizado el proceso en ellas-. La Resolución de la DGEP $N^{\circ} 35 / 07$, en su texto no garantiza una educación con énfasis en nuevas tecnologías. Se confirma una notable carencia de recursos a la vez que un vacío de capacitación efectiva y de seguimiento a la implementación. La modalidad a distancia se desarrolla con la asistencia de los estudiantes a las sedes, por lo cual no condice con la modalidad declarada sino con una Educación Media presencial nocturna y flexible.

\section{ACtores educativos}

En la EMAJA, los alumnos presentan dificultades de aprendizaje en matemática, física y química, a la vez que registran ausentismo 
y llegadas tardías. El estudiantado es mayoritariamente femenino en ambas modalidades y se presenta cuestionable que el $50 \%$ del total de los alumnos de la EMAJA corresponde a la franja etaria de la adolescencia, lo cual se reitera en la EMADJA (55\%) y los pone en condiciones etarias de insertarse en el Sistema de la Educación Media Regular. Una de las condiciones favorables de estas ofertas es que los y las docentes que se desempeñan en las mismas cuentan con certificación de docente y licenciatura. Además, se encuentran en el proceso de consolidación profesional. Este aspecto resulta propicio para desarrollar programas de capacitación continua. Los egresados -como actores de la oferta en el mercado- no tienen seguimiento sistemático.

\section{DisEÑo CURRICULAR}

Las características indican, en ambas modalidades, que el currículum es abierto, flexible, accesible, de aprendizaje permanente, con desarrollo de funciones cognitivas y profesionales. Al respecto, la realidad no favorece su desarrollo pues en la EMADJA la competencia digital que perfila a los egresados se desarrolla en instituciones que carecen de equipos informáticos para el contacto y dominio de todos los alumnos. Esto se agrava cuando deben desarrollar el "cuarto módulo" a través de la Plataforma digital sin Internet (y sin PC). Situación similar se concluye para la EMAJA, dado que para el logro de las funciones profesionales del perfil de salida, se menciona la necesidad de manejar las NTIC, pero se encuentran con las mismas carencias, aun en el Plan con énfasis en las NTIC. En cuanto a los docentes -que cuentan con la certificación correspondiente-, conocen el programa y el listado de capacidades, pero no pueden detallar los contenidos dados. Ocurre lo mismo con los tutores de la EMADJA, que indican funcionar con el "currículum entregado" (Sacristán, 2002). Se concluye que hay dificultades en la interpretación del currículum por parte de los actores en ambas modalidades investigadas. En el desarrollo curricular, se perciben vacíos de intencionalidad pedagógica y de acompañamiento a las instituciones por parte del MEC, ya que no hay evidencias de monitoreo alguno. Cabe destacar la valoración social de la oferta por parte de los actores consultados: la posibilidad de concluir sus estudios secundarios resulta altamente significativa para sus vidas, representa dignidad y la apertura a las posibilidades sociales y económicas. No se encontraron evidencias claras respecto de la calidad y la pertinencia en el desarrollo curricular de ambas ofertas. 


\section{EXPERIENCIAS DE APRENDIZAJE-ENSEÑANZA}

Las estrategias de enseñanza se caracterizan por ser predominantemente explicativas y demostrativas, con predominio del uso del pizarrón, la tiza y los ejercitarios que en la Educación a Distancia están incorporados en los textos y en la Media Alternativa los docentes los elaboran. Es altamente significativo que las tutorías de la EMADJA no se hacen por Internet (ni por la plataforma), sino presenciales, en algunos casos por teléfono o a veces por email. La línea tradicional se patentiza también en las estrategias específicamente evaluativas, pues aplican test escritos que deben aprobar las supervisiones -trámite engorroso para ambas modalidades- y control de tareas.

El abordaje de los contenidos se centra en la adquisición conceptual, donde la respuesta correcta de los ejercitarios es indicio de aprendizaje. Además, esto tiene relación con que el control de las tareas concluidas, por los participantes con ayuda de tutores, representa la valoración que permite la promoción. Se reitera la falta de acompañamiento en servicio por parte de las instancias responsables del MEC.

\section{Percepción de los actores}

De acuerdo a las percepciones registradas, se concluye que los actores perciben favorablemente a ambas modalidades por la oportunidad que ofrecen, pero no visualizan por parte del MEC la voluntad de continuar con las mismas. Asimismo, los actores tienen una percepción indudable de precariedad en los recursos para el desarrollo del currículum, percepción que eclosiona respecto del cuarto módulo de la EMADJA. Reclaman capacitación y acompañamiento del MEC para no sentirse solos y no reconocen a PRODEPA como factor favorable para el desarrollo especialmente en la EMADJA.

A partir de la información recabada se puede apreciar que las ofertas educativas estudiadas se sostienen por el alto compromiso social asumido por coordinadores y tutores y de los participantes que demuestran convicción y deseo de superación. Se resalta sobre el punto que se cuenta con las bases de un capital social en cada comunidad.

Cabe suscribir que, teniendo en cuenta los resultados de este trabajo, quedan dudas obre el carácter "a distancia" de la EMADJA, sobre cómo espera desarrollar competencia digital sin recursos tecnológicos disponibles para los procesos con los alumnos, así como sobre sus contenidos, calidad y pertinencia, y cómo se deciden las modalidades 
a implementar. Así también surgen interrogantes sobre la manera de realizar una capacitación sistemática y efectiva a los profesores de la EMAJA, y sobre qué limitaciones tuvieron los estudios de factibilidad si la implementación resulta insostenible. Estos cuestionamientos exigen la búsqueda de respuestas en nuevas investigaciones.

\section{BIBLIOGRAFÍA}

ACOSTA GONZÁLEZ, Carolina. 1996. Reforma Educacional de 1957, en Las Raíces de la Educación Paraguaya. Asunción: Gobernación del Departamento Central, pp. 65-74.

ALLIAUD, Andrea.1998. "El maestro que aprende", en Ensayos y Experiencias, Colección Psicología y Educación, Buenos Aires: Novedades Educativas, mayo-junio, Año 4 - $\mathrm{N}^{\circ}$ 23. pp. 2-17.

AQUINO, Miguel Ángel y LAFUENTE, Carlos Luis. 1990. Características y Tendencias del sistema educativo en el Paraguay (19701987). Preliminares para una aproximación crítica. Asunción: CIDSEP. BIRGIN, Alejandra y otras. 1998. La formación docente. Cultura y política. Debates y experiencias. Buenos Aires: Troquel.

CADOGAN, Miguelina. 1996. Innovaciones educacionales, en Las raíces de la educación paraguaya. Asunción: Gobernación del Departamento Central.

CASANOVA, Elsa M. 1991. Para comprender las Ciencias de la Educación. Navarra: Verbo Divino.

CEP. 1992: CONSTITUCIÓN NACIONAL, Conferencia Episcopal Paraguaya, Asunción.

CHAMORRO LEZCANO, Ubaldo. 1991. Desarrollo alternativo, en Un proyecto de desarrollo educativo alternativo para la transición hacia la democracia. Asunción: CIDE.

CONGRESO NACIONAL (1998): Ley Nº 1264 General de Educación, Asunción, El Ministerio.

CONTRERAS, José. 1997. La autonomía del profesorado. Madrid: Morata. 
DAVINI, María Cristina. 1995. La Formación docente en cuestión: Política y pedagogía. Buenos Aires: Paidós.

DE LELLA, Cayetano. 1999. Modelos y tendencias de la Formación Docente, http://www.oei.es, consultado el 08 de enero de 2005.

DELGADO, Manuel Lorenzo. 2003. Teorías curriculares. En Didáctica general: un enfoque curricular, pp. 89-107. Universidad de Granada.

DELORS, Jacques y otros. 1996. La educación encierra un tesoro. Informe de la Comisión Internacional sobre la educación para el siglo XXI... Madrid: Santillana.

DÍAZ BARRIGA, Ángel e INCLÄN, Catalina. 2001. "El docente en las reformas educativas: sujeto o ejecutor de proyectos ajenos", en Revista Iberoamericana de la Educación, $\mathrm{N}^{\mathrm{o}} 25$ enero-abril - Madrid: Organización de Estados Iberoamericanos.

FLORES OCHOA, Rafael. 2001. Docente del siglo XXI. Cómo desarrollar una práctica docente competitiva. Evaluación Pedagógica y Cognición. Bogotá: McGRAW-HILL.

GIMENOSACRISTÁN, José. 1992. Profesionalización Docente y Cambio Educativo. En Alliaud, A. y Duschtzky, L. (Compiladoras). Maestros. Práctica y Transformación Escolar. Buenos Aires: Miño y Dávila.

HERNÁNDEZ SAMPIERI, Roberto; 1997. FERNÁNDEZ COLLADO, Carlos y BATISTA LUCIO, Pilar. Metodología de la investigación. México: McGraw - Hill.

INOSTROZA DE CELIS, Gloria. 1997. La práctica, motor de la Formación Docente. Santiago: Dolmen.

KNOWLES, Malcolm, HOLTON E., Swanson. 2001 Andragogía. El aprendizaje de los adultos. Oxford University Press. México.

MACCHIAROLA DE SIGAL, Viviana. 1998 "El conocimiento práctico profesional", en Ensayos y Experiencias, Colección Psicología y Educación, Buenos Aires: Novedades Educativas, mayo-junio. Año $4 \mathrm{~N}^{\circ}$ 23. pp. 18-33.

MARTÍNEZ, Miguel. 1997. La investigación cualitativa etnográfica en educación. México: Trillas.

MEC. Programa de mejoramiento de la calidad de la educación secundaria. 1998. Aprender desde la práctica. Los traspasos necesarios para la nueva organización pedagógica. Asunción. 
MEC. 1973. Programa de desarrollo educacional Sistema de Formación Docente. Asunción.

MEC. Programa de mejoramiento educativo 1995. La Reforma pasa de grado. Módulo de capacitación. Asunción: MEC-MECES.

MEC. Viceministerio de Educación. Dirección General de Desarrollo Educativo. Dirección General de Educación Media y Técnica. 2002. Proyecto Reforma Joven. Diseño Curricular Nacional. Implementación Experimental 2002-2004. Asunción: MEC.

MEC. Consejo Asesor de la Reforma. Educativa. 1998. Avances de la Reforma Educativa. Perspectivas, estrategias y políticas de la educación paraguaya. Asunción.

MENDES DE OXILIA DÁVALOS, Tania. (Organizadora). 2002. Ciencia, Técnica y Tecnología. Reflexiones críticas. Universidad Autónoma de Asunción.

MOLINA BOGANTES, Zaida y BOLAÑO BOLAÑO, Guillermo. 1990. Introducción al Currículo. Costa Rica: EUNED.

LOPEZ, Emilio; BARAJAS ZAYAS; SARRATE CAPDEVILA; COORD. 2002. La educación de personas adultas. Reto de nuestro tiempo. Madrid. Ed. DYKINSON.

PAREDES DE MEAÑOS, Zulema. 1995. Hacia la profesionalización del docente. Buenos Aires: El Ateneo.

PÉREZ SERRANO, Gloria. 1994. Investigación cualitativa. Retos e interrogantes. II- Técnicas y análisis de datos. Madrid: La Muralla.

PÉREZ SERRANO, Gloria.1994. Investigación cualitativa. Retos e interrogantes I-Métodos. Madrid: La Muralla.

QUINTANA DE HÓRAK, Carmen. 1995. La educación escolar en el Paraguay. Apuntes para una historia. Asunción: Serie Educación: CepagSumando-Fundación En Alianza.

SALGUEIRO, Ana María. 1998. Saber docente y práctica cotidiana. Un estudio etnográfico. Barcelona: Octaedro.

SANVISENS, Alejandro. 1984. Introducción a la pedagogía. Barcelona: Barcanova.

SANZ FERNÁNDEZ, F. COORD. 2002. La Formación de agentes en educación de personas adultas. Fundamentos y marco conceptual, 
aspectos sociológicos y ocupacionales. Tomo 1 Universidad Nacional de Educación a Distancia. Madrid.

TORRES, Rosa María. 1999. "Nuevo rol docente: ¿Qué modelo de formación para qué modelo educativo?, en Boletín Proyecto Principal de Educación. No 49, pp. 38-51 agosto/99.

http://dinamicasojuegos.blogspot.com/2010/03/la-licuadora.html 